\title{
Minimizing the Maximum Flow Time in Batch Scheduling
}

\author{
Sungjin $\mathrm{Im}^{\mathrm{a}}$, Hoon $\mathrm{Oh}^{\mathrm{b}}$, Maryam Shadloo \\ ${ }^{a}$ University of California at Merced, Merced, $C A$ \\ ${ }^{b}$ Rutgers University-Camden, Camden, $N J$
}

\begin{abstract}
We consider the maximum flow time minimization problem in batch scheduling, which is a capacitated version of broadcast scheduling. In this setting, $n$ different pages of information are available at the server which receives requests from clients over time for specific pages. The server can transmit at most one page $p$ at each time to satisfy a batch of requests for the same page $p$, up to a certain capacity $B_{p}$. In this paper we give the first $(1+\epsilon)$-approximations for this problem with arbitrarily small resource augmentation, using either more capacity or more speed.
\end{abstract}

Keywords: Batch scheduling, broadcast scheduling, maximum flow time, approximation, resource augmentation.

\section{Introduction}

In batch scheduling, there is a server that stores $n$ different unit-sized pages of information. Each client submits to the server a request $\rho$ at time $r_{\rho}$ asking for a specific page $p_{\rho}$. The server can transmit at most one page $p$ at each time 5 to satisfy up to $B_{p}$ outstanding requests of the same page $p$ simultaneously; the capacity $B_{p}$ can be different for each page. Processing a batch of requests together is a popular method to increase the server's throughput. Not surprisingly, batch scheduling appears in various forms in numerous applications, not

\footnotetext{
* Corresponding author

Email addresses: sim3@ucmerced.edu (Sungjin Im), ho62@rutgers.edu (Hoon Oh), mshadloo@ucmerced.edu (Maryam Shadloo)
}

Preprint submitted to Operations Research Letters

September 29, 2016

(C) 2016. This manuscript version is made available under the Elsevier user license http://www.elsevier.com/open-access/userlicense/1.0/ 
only in server-client scheduling, but also in manufacturing lines; for pointers of the applications, see [1.

Broadcast scheduling is a special case of batch scheduling which has received considerable attention in theoretical computer science. The only difference is that in broadcast scheduling there is no limit on the number of requests the server can aggregate at a time, i.e. $B_{p}=\infty$ for all $p$. In other words, batch scheduling is a capacitated version of broadcast scheduling. However, as discussed in 2, capacities are often present in practice. For example, there could be a limit on the number of clients a server can serve at a time.

What makes batch/broadcast scheduling algorithmically challenging is that the scheduler could aggregate more requests by waiting for other requests arriv20 ing in the future for the same page. While the server can increase throughput by doing so, it makes earlier arriving requests wait longer, thereby making the clients submitting those requests unhappy. Such a tradeoff becomes more challenging in batch scheduling since the scheduler also has to factor in batch sizes. A request $\rho$ 's flow time is defined as its completion time $C_{\rho}$ minus its arrival time ${ }_{25} r_{\rho}$ and measures how long the request waits since its arrival until its completion time. When requests compete to get served earlier, a popular way of combining the flow time of individual requests is to consider flow time objectives such as total flow time or the maximum flow time.

In this paper, we study the objective of minimizing the maximum flow time, so i.e. $\max _{\rho}\left(C_{\rho}-r_{\rho}\right)$ in the batch scheduling setting. In broadcast scheduling, First-In-First-Out (FIFO) is known to be a 2-approximation [3, 4]. At each time, the algorithm FIFO transmits the page of an outstanding request with the earliest arrival time; notice that FIFO is in fact an online algorithm since it does not need to know requests arriving in the future. It was subsequently 35 shown that no online algorithms can be better than 2-competitive [3, 5]. The open question whether there exists a better than 2-approximation was recently answered in [1, which gave a PTAS using a variant of $\alpha$-point rounding and dynamic programming (DP). The work in [1] essentially closed the complexity of the problem in broadcast scheduling since the problem was already known to 
be strongly NP-hard [3].

The main goal of this paper is to understand the complexity of the maximum flow time objective in batch scheduling, which captures capacity constraints commonly appearing in practice. A recent work shows that FIFO is still 2competitive in batch scheduling [6] as it is in broadcast scheduling. Our work started from the question if there exists a better than 2-approximation in batch scheduling.

\subsection{Our Result}

Our main result is the first $(1+\epsilon)$-approximations with arbitrarily small resource augmentation. We consider two types of resources augmented, capacity and speed. In the capacity augmentation model, the algorithm is allowed to satisfy up to $(1+\delta) B_{p}$ requests of page $p$ by one transmission of page $p$ and is compared against the optimal scheduler subject to the original capacity $B_{p}$ for every $p$. In this model, if the algorithm's objective is at most $c$ times the optimum for all inputs, we say that the algorithm is a $(1+\delta)$-capacity

${ }_{55} c$-approximation. We believe that capacity augmentation model is reasonable since capacities are specified only approximately in practice when capacities are large - if all capacities are constants, we obtain a PTAS without any resource augmentation; see Section 2.2 .

In the speed augmentation model, both the algorithm and the optimal scheduler are subject to the same capacities, but the algorithm is given an extra speed. If it is given $1+\delta$ speed, it is allowed to make one additional transmission than the optimal scheduler in every $\lfloor 1 / \delta\rfloor$ time steps. In this model, we say the algorithm is a $(1+\delta)$-speed $c$-approximation if the algorithm's objective is at most $c$ times the optimum for all inputs. Speed augmentation is widely considered in 65 the scheduling literature [7].

Theorem 1. Let $m$ denote the number of requests. For minimizing the maximum flow time in batch scheduling, for any $\epsilon>0$ and $\delta>0$, we have the following approximations: 
1. [Section 2.3] a $(1+\delta)$-capacity $(1+\epsilon)$-approximation with running time $m^{O\left(\frac{1}{\epsilon^{4} \delta^{2}}\right)} ;$ and

2. [Section 2.4] a $(1+\delta)$-speed $(1+\epsilon)$-approximation with running time $m^{O\left(\frac{1}{\epsilon^{3} \delta} \cdot \log (1 /(\epsilon \delta))\right)}$.

We also show how to obtain a quasi-polynomial time approximation scheme (QPTAS) without using any extra resources in Section 2.1. Currently, we do not know how to obtain a true PTAS, which we leave as an open problem.

\subsection{Overview of our Approach}

At a high level, we closely follow the PTAS framework used in [1] for broadcast scheduling, which combines DP and a variant of $\alpha$-point rounding. We discuss how we modify each part to obtain our result in batch scheduling. We first discuss the rounding part. The rounding part is used when the optimum, opt is large, say opt $\geq \Omega(\log m)$ where $m$ is the number of requests. A standard linear programming (LP) used in broadcast scheduling is the following: variable $x_{p, t}$ denotes how much page $p$ is transmitted at time $t$, and we need constraints that (i) page $p$ must be transmitted within opt time steps after every time the page is requested, and (ii) at most one page can be transmitted at each time. In batch scheduling, we need to add more constraints to factor in capacity constraints. For every page $p$ and every interval $I=\left[t_{1}, t_{2}\right]$, we ensure that (i') at least $\left\lceil m_{p, I} / B_{p}\right\rceil$ transmissions are made for page $p$ during $\left[t_{1}, t_{2}+\right.$ opt $]$, where $m_{p, I}$ is the number of requests made during $I$ for page $p$. This new constraint, together with (ii), turns out to be necessary and sufficient conditions for a feasible integral solution to correspond to a schedule with the maximum flow time at most opt.

We use the same variant of the $\alpha$-point rounding used in [1. We give a quick overview of the rounding scheme explaining how it works well with the new LP constraint. After solving the LP, we obtain a fractional solution $\left\{x_{p, t}\right\}$ and would 95 like to round it. A standard $\alpha$-point rounding picks a random value $\alpha_{p}$ from $[0,1]$ uniformly and independently for each page $p$, then attempts to transmit page $p$ at the first time $t$ when the accumulative transmission of page $p$, i.e. $\sum_{t^{\prime} \leq t} x_{t^{\prime} p}$ becomes greater than $\alpha_{p}$ plus each non-negative integer. Note that a 
new transmission of page $p$ is made before the LP solution accumulates another unit of transmissions of page $p$. Hence due to the constraint (i), we obtain a temporary schedule with the maximum flow time at most opt. However, the temporary schedule may be infeasible since it may make too many transmissions during a short interval, which translates into a large increase of the objective when it is converted into a feasible schedule. Roughly speaking, a large number of pages/random variables results in a large variance in congestion. To overcome this issue, 1] partitioned pages into $O$ (opt) groups and used only one random variable of the maximum value at most 1 for each group, therefore was able to have a small congestion over all intervals w.h.p. The new rounding kept the key property that a new transmission of page $p$ is made before the LP solution accumulates another unit of transmissions of page $p$. Thus, the new constraint (i') we use for batch scheduling ensures that the rounding scheme makes enough transmissions in the temporary schedule while using a small number of random variables. The rounding can be de-randomized using the method of pessimistic estimators [8].

We now discuss the DP part which is used when opt $=O(\log m)$. It is easy to see that in an optimal solution, the number of distinct pages of requests alive at a time is at most opt. Using this observation, if $B_{p}=O(1)$ for all $p$, one can obtain an optimal schedule via a DP that keeps track of the number of outstanding requests for each page. In the capacity augmentation model, by adding some dummy requests and using an appropriate scaling, we reduce the general problem to the case where all capacities are constants. In the speed augmentation model, we use a different idea. We make an extra transmission of page $p$ before we have too many possibilities for the number of alive requests of the page $p$ - the transmission is used to simplify the number. Here we carefully decide which pages to transmit using extra speed since we are allowed to make only one extra transmission in every $1 / \delta$ time steps. 


\subsection{Related Work}

In batch scheduling, [6] studied online algorithms for flow time objectives. Specifically, 6] showed $O(1)$-speed $O(1)$-capacity $O(1)$-competitive algorithms

130

As mentioned before, [6] also showed that FIFO is 2-competitive for the maximum flow time objective. For the best offline results on flow time objectives in broadcast scheduling, see [9, 1].

\subsection{Notation and Organization} In other words, any 'reasonable' algorithm can complete all requests by time $T$. As observed in [1, one can assume w.l.o.g. that $T=O\left(m^{2}\right)$. This is because if there is an idle time period of length more than $m$, one can break the instance into two disjoint instances since the earlier arriving requests can be satisfied algorithms with running time polynomial (or quasi-polynomial) in $m$ and $T$, which will imply the desired results.

For notational convenience, we will use a model that is slightly different from but equivalent to the previously studied models. At each time, first a set of requests arrive, and then a page is transmitted to satisfy requests that have arrived but haven't been satisfied/completed. Note that in this model a request may have flow time 0 . This is not a problem in the study of the maximum flow time objective since we can solve the problem optimally when the optimum is a constant; see Section 2.1. Our algorithm can be easily adapted to the previously studied models.

Consider an arbitrary schedule. We let $A_{t}$ denote the set of requests alive at time $t$. When we say that a request $\rho$ is alive at time $t$, we mean that it is just after we make a transmission at the time. We say that a page $p$ is alive at time $t$ if there is a request in $A_{t}$ for the page. For a set $R$ of requests, let $P(R)$ denote the pages requested by a request in $R$. Thus, $P\left(A_{t}\right)$ refers to the set of pages alive at time $t$. Let $R_{(\cdot)}$ denote the set of requests satisfying the condition 
specified in the subscript. For example, $R_{r \leq t}$ is the set of requests arriving by time $t$.

Using a standard binary search we can assume w.l.o.g. that we know the opt $=\Omega\left(\frac{1}{\epsilon^{3}} \log T\right)$ in Sections 2 and 3 , respectively.

\section{Case opt $=O\left(1 / \epsilon^{3}\right) \log T$}

In this section we handle the case when opt $=O\left(1 / \epsilon^{3}\right) \log T$ using the (DP). We first study simpler cases when opt $=O(1)$ or $\max _{p} B_{p}=O(1)$ as a warm-up

\subsection{Warm-up: opt $=O(1)$}

We show that we can solve the problem optimally when opt $=O(1)$. We say that a set of requests alive at time $t, A_{t}$, is achievable if there is a feasible schedule up to time $t$ where all requests in $\mathcal{R}_{r \leq t}$, except exactly $A_{t}$, are satisfied by time $t$ and have flow time at most opt, and $A_{t} \subseteq R_{t-\mathrm{opt}<r \leq t}$; requests arriving 175 by time $t$-opt must be satisfied by time $t$. Clearly, it must be the case that $\left|P\left(A_{t}\right)\right| \leq$ opt since we have to satisfy all requests in $A_{t}$ within opt time steps. Let $\mathcal{A}_{t}$ be a collection that includes all possible $A_{t}$. We want to bound $\left|\mathcal{A}_{t}\right|$. By expressing $A_{t}$ as the number of requests for each page in $P\left(A_{t}\right)$, we have that $\left|\mathcal{A}_{t}\right| \leq m^{2 \text { opt }}$. This is because $\left|P\left(A_{t}\right)\right| \leq$ opt, each page in $P\left(A_{t}\right)$ has at most $m$ possibilities, and there can be at most $m$ alive requests for each page $p$.

We construct a DP table with entries $\left\{\mathrm{DP}\left(t, A_{t}\right)\right\}_{t, A_{t} \in \mathcal{A}_{t}}$ where $A_{t} \subseteq R_{t-\mathrm{opt}<r \leq t}$; note that $A_{t}$ can only include requests that have been waiting for less than opt times steps since their arrival. From the above discussion, the table size is manageable, at most $m^{2 \mathrm{opt}} \cdot T$. Each entry $\operatorname{DP}\left(t, A_{t}\right)$ has value either true or false, 
185 as follows: $\operatorname{DP}\left(t, A_{t}\right)$ is true if and only if there is an entry $\operatorname{DP}\left(t-1, A_{t-1}\right)$ with true value such that we can obtain $A_{t}$ by removing up to $B_{p}$ requests of a certain page $p$ from $A_{t-1} \cup R_{r=t}$. The time needed to fill out the entire table is only $\operatorname{poly}(m)$ factor larger than the DP table size. Notice that there is a

\subsection{Warm-up: $B:=\max _{p} B_{p}=O(1)$}

In this case we make another observation to reduce the number of possibilities of $P\left(A_{t}\right)$ - our argument in the previous section only gives a very loose upper bound of $m^{\text {opt }}$, which is not useful since opt is no longer a constant. Note that $\left(\begin{array}{c}B \text { opt+opt } \\ \text { opt }\end{array}\right) \leq 2^{(B+1) \text { opt }}$; see Proposition 1 . Therefore, we have $\left|\mathcal{A}_{t}\right| \leq 2^{(B+3) \text { opt }}$. Since $B=O(1)$ and opt $=O(\log m)$ for any fixed $\epsilon$, we have a poly-sized DP table which we can complete in polynomial time.

Proposition 1. Let $z_{1}, z_{2}, \ldots, z_{k}$ be variables which can take non-negative in210 $\left(\begin{array}{c}L+k \\ k\end{array}\right) \leq 2^{L+k}$ 


\subsection{General Case under Capacity Augmentation}

In this section we give a $(1+\epsilon)$-approximation for the case opt $=O\left(\frac{1}{\epsilon^{3}} \log T\right)$ assuming that our algorithm can handle $(1+\delta)$ larger batches than the optimal scheduler. Our main idea is to cluster requests of the same page to reduce the general case to the constant $B$ case, which we already know how to solve. To streamline our presentation, we assume that $1 / \epsilon, 1 / \delta$ and $\epsilon$ opt are all integers; the last assumption is w.l.o.g. since we can solve the case when opt $=O(1)$ optimally.

We take the following two preprocessing steps to simplify the instance. We say a time $t$ is a grid time if it is an integer multiple of $\epsilon$ opt.

1. Shift each request $\rho$ 's arrival time to the right to the closest grid time, i.e. $\epsilon$ opt $\left\lceil\frac{r_{\rho}}{\epsilon \text { opt }}\right\rceil$.

2. Consider each page $p$ with $B_{p} \geq \frac{2}{\delta \lambda}$ where $\lambda:=\delta \epsilon / 4$ : Let $B_{p}^{\prime}$ be the largest integer no greater than $B_{p}$ that is an integer multiple of $1 / \lambda$; note that $B_{p}^{\prime} \geq \frac{2}{\delta \lambda}$. Round up $m_{p, t}$, the number of requests arriving for each page $p$ at time $t$, to the nearest integer multiple of $\lambda B_{p}^{\prime}$, i.e. $\lambda B_{p}^{\prime}\left\lceil\frac{m_{p, t}}{\lambda B_{p}^{\prime}}\right\rceil$. Replace $B_{p}$ with $\frac{4(1+\delta)}{\epsilon \delta}$ and $m_{p, t}$ with $\left\lceil\frac{m_{p, t}}{\lambda B_{p}^{\prime}}\right\rceil$ (scaling down $B_{p}$ and $m_{p, t}$ ).

Note that we have $B:=\max _{p} B_{p} \leq \frac{8}{\epsilon \delta^{2}}$ after the above modifications. Recall that $T=O\left(\mathrm{~m}^{2}\right)$ and we have shown that the DP is of size at most $T \cdot 2^{(B+3) \text { opt }}=$ $m^{O\left(\frac{1}{\epsilon^{4} \delta^{2}}\right)}$. This shows the running time in the capacity augmentation model claimed in Theorem 1 .

It now remains to show that the above reduction is valid. It is obvious that the first modification increases the optimum by at most $\epsilon$ opt since delaying the entire optimal schedule by $\epsilon$ opt is a feasible schedule for the modified instance. For notational convenience, assume that there is a schedule with the maximum flow time at most opt for this modified instance; this is w.l.o.g. due to the asymptotic bounds claimed in the theorem.

We now show that even after we increase $m_{p, t}$ for some pages in the second modification, the optimal objective doesn't increase when strengthened with $(1+\delta)$ capacity augmentation. The schedule will remain the same except that 
it may have to satisfy more requests for page $p$ by one transmission, namely up to $(1+\delta) B_{p}^{\prime}$. To see this, consider a transmission of page $p$. Say the transmission is made at time $t$. The transmission satisfies some requests arriving during ${ }_{245}[t-\mathrm{opt}, t]$. Here we let the transmission satisfy the extra requests of page $p$ we added during $[t-\mathrm{opt}, t]$. Hence in the worst case, the number of requests it should satisfy is at most $\lambda B_{p}^{\prime}\left(\frac{1}{\epsilon}+1\right)+B_{p} \leq \frac{\delta}{2} B_{p}^{\prime}+B_{p}^{\prime}+1 / \lambda \leq(1+\delta) B_{p}^{\prime}$; there are at most $1 / \epsilon+1$ grid times during the interval, requests arrive only at grid times after the first modification, and at most $\lambda B_{p}^{\prime}$ requests of page $p$ are added at each grid time. Further, note that since we only increased $m_{p, t}$, a schedule for the modified instance can be easily translated into one for the original instance without increasing the maximum flow time.

Finally, we briefly discuss why we can replace $B_{p}$ with $\frac{4(1+\delta)}{\epsilon \delta}$ for some pages $p$. Note that the number of requests of page $p$ arriving at time $t, m_{p, t}$ and $255(1+\delta) B_{p}^{\prime}$ are both integer multiples of $\lambda B_{p}^{\prime}$. What this means is that the number of requests for page $p$ alive at each time is always an integer multiple of $\lambda B_{p}^{\prime}$. Hence we can scale down the numbers by a factor of $\lambda B_{p}^{\prime}$; notice that $\frac{(1+\delta) B_{p}^{\prime}}{\lambda B_{p}^{\prime}}=\frac{4(1+\delta)}{\epsilon \delta}$.

\subsection{General Case under Speed Augmentation}

In this section we give a $(1+\epsilon)$-approximation for the case opt $=O\left(\frac{1}{\epsilon^{3}} \log T\right)$ assuming that our algorithm can make one additional transmission in every $1 / \delta$ time steps. Assume that $\epsilon, \delta \leq 1 / 10$. For simplicity, assume that $1 / \delta$, $1 / \epsilon$, and $\epsilon \delta$ opt are all integers. We also assume w.l.o.g. that requests arrive and are satisfied only at grid times (integer multiples of $\epsilon \mathrm{opt}$ ), and at most $\epsilon$ opt transmissions are made at each grid time. As observed in [1, this will increase the approximation factor by a factor of at most $(1+2 \epsilon)$. We assume that there is a schedule with the maximum flow time opt; this is w.l.o.g. due to the asymptotic bound claimed in Theorem 1. Since we have $(1+\delta)$-speed augmentation, we are allowed to make up to $(1+\delta) \epsilon$ opt transmissions at each grid time.

In the speed augmentation model, we can't add a small number of requests 
at every grid time for each alive page to simplify the instance as we did in the capacity augmentation model. This is because even an extra request may force the algorithm to make one more transmission, but we only have $(1+\delta)$-speed

The key idea is to make an extra transmission of page $p$ before the number of possibilities of $n_{p, t}$ becomes unaffordable. Here $n_{p, t}$ denotes the number of requests of page $p$ that are alive at time $t$ in a certain schedule in consideration; this is different from $m_{p, t}$ which denotes the number of requests arriving at time $t$ for page $p$. When we make a transmission of page $p$ at time $t$ using speed augmentation (this is one of the $\epsilon \delta$ opt extra transmissions made at each grid time), we ensure that $B_{p} \mid n_{p, t}\left(n_{p, t}\right.$ is divisible by $\left.B_{p}\right)$ after the transmission. At each time, at most one transmission will be made using speed augmentation for each page. Thus we can assume that at each grid time we first make $\epsilon$ opt 'regular' transmissions and then at most $\epsilon \delta$ opt 'extra' transmissions. A regular transmission of a page is used to satisfy as many requests of the page as possible in FIFO fashion. In contrast, we use an extra transmission of page $p$ only to have $B_{p} \mid n_{p, t}$.

We now describe how we find the extra pages we will transmit using speed augmentation. We will mark some pages at each grid time and will make an extra transmission of page $p$ at time $t$ if page $p$ is marked at the time. To describe the marking procedure, we need to define $W_{p}$ for each page $p$. We say that two requests $\rho$ and $\rho^{\prime}$ for the same page $p$ are adjacent if $r_{\rho} \neq r_{\rho^{\prime}}$ and no requests arrive for page $p$ between times $r_{\rho}$ and $r_{\rho^{\prime}}$. We can assume w.l.o.g. that any two adjacent requests $\rho$ and $\rho^{\prime}$ for the same page arrive within opt time steps since otherwise, no transmission of page $p$ can satisfy requests arriving no later than $\rho$ and those arriving no earlier than $\rho^{\prime}$ simultaneously; here we assumed $r_{\rho}<r_{\rho^{\prime}}$. For each page $p$, define $W_{p}:=\left[\min \left\{r_{\rho}: p_{\rho}=p\right\}, \max \left\{r_{\rho}: p_{\rho}=p\right\}+\right.$ opt $]$, which we call page $p$ 's window. Notice that we can assume w.l.o.g. that the optimal schedule transmits page $p$ only during $W_{p}$. We are now ready to describe the marking procedure. 
Marking procedure: Consider times in increasing order. There is a queue $\mathcal{Q}_{t}$ containing all pages $p$ such that $t \in W_{p}$. Each page $p \in \mathcal{Q}_{t}$ is associated with its most recent marking time $u_{p}$. At each grid time $t, u_{p} \leftarrow t$ if the page hasn't been requested yet. At each grid time $t$, we choose up to $\delta \epsilon$ opt pages with the smallest $u_{p}$ and let $u_{p} \leftarrow t$ for such pages $p$.

Lemma 1. Let $\tau_{p, t}$ denote the latest (grid) time no later than $t$ when $p$ is marked. For any time $t$ and any page $p$ such that $t \in W_{p}$, we have $t-3 o p t / \delta<$ $\tau_{p, t} \leq t$.

Proof. For the sake of contradiction, let $t^{*}$ be the first time $t$ such that for some page $p, t \in W_{p}$ and page $p$ is not marked at any time during $I=(t-3 \mathrm{opt} / \delta, t]$. Note that we make 3opt markings during $I$. A crucial observation is that no page is marked more than once during $I$ since when a page $q$ is marked, its latest marking time $u_{q}$ will become greater than $u_{p}$ and page $p$ is not marked during $(t-3 \mathrm{opt} / \delta, t]$. Let $Q$ denote the set of pages marked during $I$. Note that $|Q| \geq 3$ opt. Let $t_{1}:=t-3 \mathrm{opt} / \delta$ be the start time of $I$. Note that for any $q \in Q$, $t_{1} \in W_{q}$. This is because otherwise either $q$ hasn't been requested till time $t_{1}$, thus $u_{q}$ is greater than $u_{p}$, or all requests of page $q$ must have been completed before time $t_{1}$. In either case, $q \notin Q$. Hence any page $q$ in $Q$ is requested 320 during $\left[t_{1}-\mathrm{opt}, t_{1}\right]$, meaning that all pages in $Q$ must be transmitted during $\left[t_{1}-\mathrm{opt}, t_{1}+\mathrm{opt}\right]$ at least once. Since at most $(1+\delta)(2+\epsilon)$ opt transmissions can be made during the interval and $|Q| \geq 3$ opt, we have a contradiction.

The following lemma implies that there exists a $(1+\delta)$-speed schedule with a good structural property that is as good as the optimal schedule. The proof immediately follows by applying Lemma 1 to the optimal schedule.

Lemma 2. There exists a $(1+\delta)$-speed schedule with the maximum flow time at most opt where for any $p$ and $t$, if $n_{p, t}>0$, then there is a time $t-3 o p t / \delta<$ $t^{\prime} \leq t$ such that $B_{p} \mid n_{p, t^{\prime}}$. Further, at each grid time $t$, the schedule makes an extra transmission of page $p$ if and only if $p$ is marked at the time $t$. 

which we can reduce using Lemma 2

Lemma 3. For any $t,\left|\mathcal{A}_{t}\right| \leq\left(\frac{144}{\epsilon^{2} \delta^{2}} 2^{7 / \delta}\right)^{\text {opt }}$.

Proof. Fix a time $t$ and $p \in P\left(A_{t}\right)$. By Lemma 1. $\tau_{p, t}$ can have at most $\frac{3}{\epsilon \delta}$ different values. Let $\tau_{p, t}^{\prime}$ be the latest time $t^{\prime}$ no later than $t$ such that $B_{p} \mid n_{p, t^{\prime}}$. 335 Note that $\tau_{p, t} \leq \tau_{p, t}^{\prime}$. By definition of $\tau_{p, t}^{\prime}$, we know that at least one request is alive for page $p$ at each time during $\left(\tau_{p, t}^{\prime}, t\right]$. This implies that every transmission of page $p$ made during $\left(\tau_{p, t}^{\prime}, t\right]$ satisfies exactly $B_{p}$ requests, meaning that $n_{p, \tau_{p, t}^{\prime}}$ and $z_{p}$, the number of transmissions of page $p$ made during $\left(\tau_{p, t}^{\prime}, t\right]$ determines $n_{p, t}$.

Let $N(\cdot)$ denote the number of possibilities of what is inside the parentheses. We first upper bound $N\left(P\left(A_{t}\right)\right)$. Note that $P\left(A_{t}\right) \subseteq P\left(R_{t-\mathrm{opt}<r \leq t}\right)$, and $\left|P\left(R_{t-\mathrm{opt}<r \leq t}\right)\right| \leq 2(1+\delta)$ opt $\leq 4$ opt. Hence we have $N\left(P\left(A_{t}\right)\right) \leq 2^{4 \text { opt }}$. Now we bound $N\left(\left\{\tau_{p, t}^{\prime}\right\}_{p \in P\left(A_{t}\right)}\right)$ for each fixed $P\left(A_{t}\right)$. As mentioned before, each $\tau_{p, t}^{\prime}$ can have at most $\frac{3}{\epsilon \delta}$ different values. Hence, $N\left(\left\{\tau_{p, t}^{\prime}\right\}_{p \in P\left(A_{t}\right)}\right) \leq\left(\frac{3}{\epsilon \delta}\right)^{2 \text { opt }}$ for tions since we have to decide the $\epsilon(1+\delta)$ opt transmissions we make at each grid time. Let $P_{t}$ denote the multi-set of pages we transmit by regular transmissions at time $t$. Let $t^{\prime}=t-\epsilon$ opt. Each entry $\operatorname{DP}\left(t, A_{t}\right)$ is set to true if and only if there are $A_{t^{\prime}}$ and $P_{t}$ such that $A_{t}$ is achieved from $A_{t^{\prime}} \cup R_{r=t}$ by transmitting pages in the multi-set $P_{t}$ and rounding down $n_{p, t}$ to the nearest integer multiple of $B_{p, t}$ for each page $p$ that is marked at time $t$. From the proof of Lemma 3 it is easy to see that the number of possibilities of $P_{t}$ is upper bounded by $2^{4 \mathrm{opt}} \cdot 2^{7 \mathrm{opt} / \delta} \leq 2^{8 \mathrm{opt} / \delta}$; here we did not optimize this loose bound. Therefore, we conclude that the running time of the DP is at most $\left(\frac{144}{\epsilon^{2} \delta^{2}} 2^{15 / \delta}\right)^{\mathrm{opt}}$, in which 

on opt. An elementary calculation gives the running time claimed in Theorem 1 for the speed augmentation model.

\section{Case opt $\geq\left(1 / \epsilon^{3}\right) \log T$}

We consider the following integer programming problem that determines if there is a feasible schedule with the maximum flow time of at most opt. Let $m_{p, I}:=\sum_{t \in I} m_{p, t}$. See Section 2.4 for the definition of $m_{p, t}$ and $W_{p}$.

$$
\begin{array}{cl}
\sum_{t_{1} \leq t \leq t_{2}+\text { opt }} x_{p, t} \geq\left\lceil\left(m_{p,\left[t_{1}, t_{2}\right]} / B_{p}\right)\right\rceil & \forall p, t_{1} \leq t_{2} \in[T] \\
\sum_{p} x_{p, t} \leq 1 & \forall t \in[T] \\
x_{p, t}=0 \quad \forall p, t \notin W_{p} ; & x_{p, t} \in\{0,1\} \quad \forall p, t \in[T]
\end{array}
$$

Lemma 4. The above IP exactly captures the maximum flow time problem in batch scheduling. In other words, there exists an one-to-one mapping between a feasible solution to the IP and a feasible schedule with the maximum flow time at most opt.

Proof. We will first discuss what each constraint captures. The first constraint ensures that all requests must be completed within opt time steps. The second constraint states that at most one page can be transmitted at each time. The third implies that no transmission is made if it cannot be used to satisfy a request within opt time steps.

The claimed one-to-one mapping is naturally defined from what $x_{p, t}$ is meant to encode: $x_{p, t}=1$ if and only if page $p$ is transmitted at time $t$, and requests for the same page are satisfied in FIFO order. To establish the one-to-one mapping, first consider an arbitrary schedule $\sigma$ with the maximum flow time at most opt. Then, we know that to satisfy requests arriving during $\left[t_{1}, t_{2}\right]$ for page $p$, we need to transmit page $p$ at least $\left\lceil m_{p,\left[t_{1}, t_{2}\right]} / B_{p}\right\rceil$ times during $\left[t_{1}, t_{2}+\right.$ opt $]$, which is exactly what the first constraint says. It is obvious that $\left\{x_{p, t}\right\}$ derived from ${ }_{380} \sigma$ satisfies other constraints. 
We now show the opposite direction. Let $\left\{x_{p, t}\right\}$ be an arbitrary feasible solution to the IP and $\sigma$ the schedule corresponding to the IP solution. We show that every request has flow time at most opt in $\sigma$. Let $C_{\rho}$ denote the time when $\rho$ is completed/satisfied. For the sake of contradiction, let $\rho^{*}$ be a request with flow time greater than opt, that has the earliest completion time. Let $p=p_{\rho^{*}}$. Let $t^{\prime}$ be the latest time $t$ before $C_{\rho^{*}}$ such that a transmission of page $p$ at time $t$ satisfied less than $B_{p}$ requests; if the time doesn't exist, let $t^{\prime}$ be the first time when a request arrives for page $p$, minus 1 . Clearly, any request arriving by time $t^{\prime}$ is satisfied by time $t^{\prime}$ and has flow time at most opt by the definition of $\rho^{*}$. We use the first constraint with $t_{1}=t^{\prime}+1$ and $t_{2}=r_{\rho^{*}}$. We know that $m_{p,\left[t_{1}, t_{2}\right]}$ requests arrive during $\left[t_{1}, t_{2}\right]$ for page $p$,

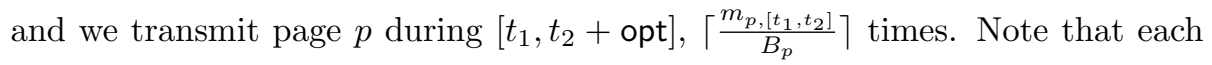
transmission satisfies exactly $B_{p}$ requests by the definition of $t^{\prime}$ and $t_{1}$. This means that $\rho^{*}$ must be satisfied by time $t_{2}+$ opt, which is a contradiction to the assumption that $\rho^{*}$ has flow time greater than opt.

We relax the IP by replacing the last constraints with $x_{p, t} \geq 0$. Let's call the resulting LP as LP MaxFlow. We solve LP MaxFlow and let $x_{p, t}^{*}$ denote the optimal fractional solution. As mentioned, we use the rounding scheme developed in [1]. For completeness, we summarize the rounding scheme and explain how it works for batch scheduling.

Pages are partitioned into groups $\mathcal{G}$ such that all pages in the same group $g \in \mathcal{G}$ have disjoint windows. It was shown in Lemma 2.2 in [1] that 2opt groups are enough for this partition using the structural property that any two 'adjacent' requests of a page $p$ must be made within opt -1 time steps. Here, we get a slightly looser bound, $|\mathcal{G}| \leq 2$ opt +1 because in this paper we allow a request to be eligible to be satisfied upon its arrival, hence we can only have that any two 'adjacent' requests of a page $p$ must be made within opt time steps; see the definition of $W_{p}$ in Section 2.4. However, the remaining analysis in [1] remains exactly the same since constants were not optimized there.

For each group $g \in \mathcal{G}$, define a cumulative amount of transmission made by 
the fractional solution. Formally, define $y_{g, t}^{*}:=\sum_{p \in g} \sum_{t^{\prime} \leq t} x_{p, t^{\prime}}^{*}$. Now for each group $g$, pick $\alpha_{g}$ from $[0,1]$ uniformly at random. We will obtain a tentative schedule $\sigma_{\text {temp }}$ and transform it to $\sigma_{\text {final }}$. In $\sigma_{\text {temp }}$, all requests are satisfied within opt time steps, and more than one transmission can be made at a time. In $\sigma_{\text {temp }}$, for each group $g$, we transmit a page $p \in g$ at the earliest time $t$ when $y_{g, t}^{*}-\alpha_{g} \geq k$ for each non-negative integer $k$. The grouping is done so that for any time $t$, there is at most one page $p \in g$ with $x_{p, t}^{*}>0$. Then we transform $\sigma_{\text {temp }}$ into the final feasible schedule $\sigma_{\text {final }}$ by rescheduling transmissions in FIFO fashion. More precisely, we keep the order of transmissions, breaking ties arbitrarily, and ensure that every transmission should be made no earlier in $\sigma_{\text {final }}$ than $\sigma_{\text {temp }}$. Also it is ensured that $\sigma_{\text {final }}$ make at most one transmission at each time. We will show that the integral solution corresponding to $\sigma_{\text {final }}$ is feasible to the IP with opt replaced with $(1+6 \epsilon)$ opt w.h.p., meaning that it represents a feasible schedule with the maximum flow time at most $(1+6 \epsilon)$ opt.

Proposition 2 immediately follows by observing that the LP solution $x^{*}$ transmits page $p$ by at least $\left\lceil\frac{m_{p,\left[t_{1}, t_{2}\right]}}{B_{p}}\right\rceil$ units during $\left[t_{1}, t_{2}+\mathrm{opt}\right]$, and the rounding scheme guarantees to make one transmission of page $p$ before the fractional solution $x^{*}$ accumulates another unit of transmission of the page $p$.

Proposition 2. The IP solution corresponding to $\sigma_{\text {temp }}$ satisfies the constraint (1).

We complete the analysis by showing that w.h.p. no request's flow time increases too much in the transformation from $\sigma_{\text {temp }}$ into $\sigma_{\text {final }}$. It was shown in [1] that the increase in the objective is upper-bounded by the global overflow, $\max _{I} O F(I)$ where $Q_{\text {temp }}(I)$ is the number of transmissions made in $\sigma_{\text {temp }}$ during time interval $I$ and $O F(I):=\max \left\{Q_{\text {temp }}(I)-|I|, 0\right\}$. In words, $O F(I)$ measures how many more transmissions are attempted to make during $I$ than are allowed.

We now upper-bound the overflow during each fixed interval $I$ as follows. The proof uses the facts that there are $O(\mathrm{opt})$ random variables that contribute 440 to the overflow, and each random variable corresponding to each group can 
contribute to the overflow $O F(I)$ by at most one. More precisely, for each group $g$, depending on the value of $\alpha_{g}$, we make transmission of pages from $g$ either $\left\lceil\sum_{t \in I} \sum_{p \in g} x_{p, t}^{*}\right\rceil$ or $\left\lfloor\sum_{t \in I} \sum_{p \in g} x_{p, t}^{*}\right\rfloor$ times during $I$ - in the former case, $g$ contributes to $O F(I)$ by $\left\lceil\sum_{t \in I} \sum_{p \in g} x_{p, t}^{*}\right\rceil-\sum_{t \in I} \sum_{p \in g} x_{p, t}^{*}$. Since we use the same rounding method, we get the same upper bound on the overflow which was shown in [1].

Lemma 5 ([1]). Suppose that opt $\geq \frac{1}{\epsilon^{3}} \log T$. Then for any interval $I, \operatorname{Pr}[O F(I) \geq$ $6 \epsilon o p t] \leq 1 / T^{3}$.

Finally, by applying a union bound over all possible intervals (at most $T^{2}$ ), we have that $\max _{I} O F(I)$ is at most 6єopt w.h.p. As mentioned before, this implies that each transmission moves to the right by at most $6 \epsilon$ opt time steps from $\sigma_{\text {temp }}$ to $\sigma_{\text {final }}$. This also implies that all the IP constraints are satisfied when opt is replaced with $(1+6 \epsilon)$ opt. Thus, we have a randomized algorithm that yields a feasible schedule with the maximum flow time at most $(1+6 \epsilon)$ opt with probability least $1-1 / T$. The algorithm can be easily derandomized using the method of pessimistic estimators [8, 1]. By scaling $\epsilon$, we have Theorem 1 when opt $=\Omega\left(\frac{1}{\epsilon^{3}} \log T\right)$, with no resource augmentation. The running time is clearly polynomial in $m$, and is dominated by that of the DPs in Section 2 .

\section{Acknowledgements} and CCF-1617653. The authors greatly thank the anonymous reviewer for the numerous comments which helped significantly improve the presentation of this paper.

\section{References}

[1] S. Im, M. Sviridenko, New approximations for broadcast scheduling via variants of $\alpha$-point rounding, in: ACM-SIAM Symposium on Discrete Algorithms, 2015, pp. 1050-1069. 
[2] A. Bar-Noy, S. Guha, Y. Katz, J. S. Naor, B. Schieber, H. Shachnai, Throughput maximization of real-time scheduling with batching, ACM Trans. Algorithms 5 (2) (2009) 18:1-18:17.

[3] J. Chang, T. Erlebach, R. Gailis, S. Khuller, Broadcast scheduling: Algorithms and complexity, ACM Trans. Algorithms 7 (4) (2011) 47.

[4] C. Chekuri, S. Im, B. Moseley, Online scheduling to minimize maximum response time and maximum delay factor, Theory of Computing 8 (1) (2012) $165-195$.

[5] C. Chekuri, A. Gal, S. Im, S. Khuller, J. Li, R. M. McCutchen, B. Moseley, L. Raschid, New models and algorithms for throughput maximization in broadcast scheduling - (extended abstract), in: Workshop on Approximation and Online Algorithms, 2010, pp. 71-82.

[6] S. Im, B. Moseley, Brief announcement: online batch scheduling for flow objectives, in: ACM Symposium on Parallelism in Algorithms and Architectures, 2013, pp. 102-104.

[7] B. Kalyanasundaram, K. Pruhs, Speed is as powerful as clairvoyance, J. ACM 47 (4) (2000) 617-643.

[8] P. Raghavan, Probabilistic construction of deterministic algorithms: Approximating packing integer programs, J. Comput. Syst. Sci. 37 (2) (1988) $130-143$.

[9] N. Bansal, M. Charikar, R. Krishnaswamy, S. Li, Better algorithms and hardness for broadcast scheduling via a discrepancy approach, in: ACMSIAM Symposium on Discrete Algorithms, 2014, pp. 55-71. 